2015

\title{
Continuous wave cavity ring-down spectroscopy for velocity distribution measurements in plasma
}

D. McCarren

E. Scime

Follow this and additional works at: https://researchrepository.wvu.edu/faculty_publications

\section{Digital Commons Citation}

McCarren, D. and Scime, E., "Continuous wave cavity ring-down spectroscopy for velocity distribution measurements in plasma" (2015). Faculty Scholarship. 99.

https://researchrepository.wvu.edu/faculty_publications/99

This Article is brought to you for free and open access by The Research Repository @ WVU. It has been accepted for inclusion in Faculty Scholarship by an authorized administrator of The Research Repository@ WVU. For more information, please contact ian.harmon@mail.wvu.edu. 


\title{
Continuous wave cavity ring-down spectroscopy for velocity distribution measurements in plasma
}

\author{
D. McCarren ${ }^{1,2}$ and E. Scime ${ }^{1, a)}$ \\ ${ }^{1}$ Department of Physics and Astronomy, West Virginia University, Morgantown, West Virginia 26506, USA \\ ${ }^{2}$ Lockheed Martin, Palmdale, California 93599, USA
}

(Received 5 June 2015; accepted 22 September 2015; published online 9 October 2015)

\begin{abstract}
We report the development of a continuous wave cavity ring-down spectroscopic (CW-CRDS) diagnostic for real-time, in situ measurement of velocity distribution functions of ions and neutral atoms in plasma. This apparatus is less complex than conventional CW-CRDS systems. We provide a detailed description of the CW-CRDS apparatus as well as measurements of argon ions and neutrals in a high-density $\left(10^{9} \mathrm{~cm}^{-3}<\right.$ plasma density $\left.<10^{13} \mathrm{~cm}^{-3}\right)$ plasma. The CW-CRDS measurements are validated through comparison with laser induced fluorescence measurements of the same absorbing states of the ions and neutrals. (C) 2015 AIP Publishing LLC. [http://dx.doi.org/10.1063/1.4932313]
\end{abstract}

\section{INTRODUCTION}

Recently, our research group demonstrated the feasibility of in situ ion velocity distribution measurements in a plasma using a variant of continuous wave cavity ring-down spectroscopy (CW-CRDS). ${ }^{1}$ Here, we report on significant improvements to the CW-CRDS apparatus that have enabled detailed measurements of argon ion and neutral velocity distribution function (VDF) measurements in argon plasma for a wide range of operating parameters in a helicon plasma source. While CRDS has a many decades history of use as a highly sensitive absorption technique for measuring very low concentrations of analytes in atmospheric pressure systems, ${ }^{2}$ the application of CRDS to low pressure plasmas is a relatively recent development. The interested reader is referred to some excellent reviews on the progress of CRDS..$^{3-7}$

The CW-CRDS measurement process involves the injection of narrowband laser light into an optical cavity. When a resonance condition is achieved in the cavity, the injected light is blocked by a shutter. The decay of the intensity of light in the cavity is then measured in a wavelength range around the absorption line of interest. The decay time is strongly modified by absorbers along the laser path in the cavity. Measurement of the time constant of decaying light, the cavity ring-down time, in an optical cavity consisting of two planoconcave mirrors was first used by Anderson et al. ${ }^{8}$ in 1984 as a method of accurately calculating the reflectivity of dielectric coatings. The first cavity ring-down spectroscopic measurements were made by O'Keefe and Deacon in $1988 .{ }^{2}$ In their pulsed cavity ring-down spectroscopy (P-CRDS) experiment, they measured the absorption spectrum of oxygen molecules in the optical cavity with a nitrogen-gas-pumped pulsed dye laser.

With the replacement of cumbersome pulsed dye lasers with smaller, less expensive, continuous wave $(\mathrm{CW})$ diode lasers, CW-CRDS systems have become widely used for

a)E-mail: earl.scime@mail.wvu.edu high-resolution spectroscopic measurements. ${ }^{9}$ Hodges et al. demonstrated that single mode, narrow linewidth lasers provide the maximum sensitivity for CRDS measurements. ${ }^{10,11}$ CW-CRDS is experimentally more challenging than P-CRDS, but the capability to measured detailed absorption spectra makes CW-CRDS particularly attractive for measuring the Doppler broadening and shift of absorption lines. ${ }^{12} \mathrm{CW}-\mathrm{CRDS}$ has been used as a diagnostic tool for atmospheric pressure plasma discharges, ${ }^{13}$ elemental detection, ${ }^{14}$ Hall thrusters, ${ }^{15}$ and trace metal detection with a portable system. ${ }^{16}$ The few implementations of CW-CRDS in plasmas in low-pressure environments have typically focused on target species detection and not wavelength resolved velocity distribution measurements. The CW-CRDS decay rate versus laser frequency provides a measure of the number of absorbers traveling at a speed such that the laser light is Doppler shifted to an absorption line in the ion/neutral's frame of reference. Thus, by measuring the decay times versus laser frequency, the absorber VDF is measured. CW-CRDS velocity distribution function measurements in a plasma require a narrow linewidth, tunable, $\mathrm{CW}$ laser; a high finesse optical cavity surrounding the plasma column; and, until now, a means of moving the cavity defining mirrors inside the vacuum environment.

CW-CRDS is not the only method for VDF measurement in plasmas. In low pressure plasmas, laser induced fluorescence (LIF) is commonly used to obtain spatially resolved temperatures and flow speeds of neutral and ion species through complete measurements of the VDF. ${ }^{17}$ Despite LIF's versatility and sensitivity, the technique has significant limitations. For LIF, the initial state of the absorption sequence must have a substantial density and the radiative decay of the excited state must occur at a detectable wavelength. Collision rates must also be small enough so that the absorbing and fluorescing states are not collisionally quenched. These limitations prevent the use of LIF to measure the VDFs of several species of interest and for a wide range of plasma source operating parameters. For example, helium-based plasmas are of widespread scientific and industrial interest. However, helium ions are notoriously difficult to fluoresce. Very long 
path length absorption spectroscopy measurements in our laboratory hinted at the existence of a sufficient population of absorbing helium ions, but fluctuations in the probing laser intensity resulted in very poor signal-to-noise and irreproducible results. ${ }^{18}$

Although our initial in situ CW-CRDS measurements were encouraging, the apparatus and methodology used for those measurements was overly complex, had poor signalto-noise, and the optics became damaged after only minutes of use. Here, we describe a much improved apparatus that substantially improves the signal-to-noise and shows no evidence of degradation after many hours of use. The key improvements include laser modulation with increased extinction ratio, use of a single mode fiber for beam shaping and mode control, elimination of mirror scanning to obtain resonance in the cavity, and protection of the in situ mirrors with flowing gas "curtains."

\section{THEORETICAL BACKGROUND}

In a CRDS measurement, the ring-down times, $\tau$, are determined by fitting an exponential function to the measured light intensity decay after the resonance condition is achieved and the laser light injected into the cavity is blocked by turning off or shuttering the laser. Assuming uniform absorption in the plasma, ring-down measurements as a function of laser wavelength are converted to measurements of absorption, $\alpha$, through the relationship

$$
\alpha \frac{d}{L}=\frac{1}{c \tau}-\frac{1}{c \tau_{o}},
$$

where $d$ is the length of the plasma, $L$ is the length of the cavity formed by the two mirrors, $c$ is the speed of light in vacuum, and $\tau_{o}$ is the ring-down time in the absence of plasma. Note that Eq. (1) is independent of the laser intensity. The fact that fluctuations in laser power are ignorable for CW-CRDS measurements is responsible for the much greater sensitivity of the CW-CRDS method over other absorption spectroscopy techniques.

The laser frequency dependent, dimensionless line-integrated absorption measure $S(v)$ is given by

$$
S(v)=\frac{d}{c \tau_{v}}-\frac{d}{c \tau_{o}},
$$

where $\tau_{v}$ is the ring-down time for a laser frequency of $v$. Assuming the measured absorption line shape is dominated by Doppler broadening, the absorption measure is describable with a single Gaussian function of the form

$$
S(v)=S\left(v_{o}\right) \exp \left[-\left(v-v_{o}\right)^{2} / b_{s} T_{S}\right],
$$

where $v_{o}$ is absorption frequency in the frame of the target species; $T_{\mathrm{s}}$ is the temperature of the target species; $b_{s}=2 e v_{o s}^{2} /$ $m_{s} c^{2} ; m_{s}$ is the mass of the target species, $s ; e$ is the electron charge; and $v_{o s}$ is the rest frame absorption frequency of the target species. For argon ions and neutrals, respectively,

$$
\begin{aligned}
& b_{\text {ArII }}^{-1}=0.092495 \mathrm{eV} / \mathrm{GHz}^{2}, \\
& b_{\text {ArI }}^{-1}=0.092384 \mathrm{eV} / \mathrm{GHz}^{2} .
\end{aligned}
$$

TABLE I. Ar I and Ar II transition parameters.

\begin{tabular}{lcc}
\hline \hline Parameter & Ar I & Ar II \\
\hline$g_{1}$ & 3 & 8 \\
$g_{2}$ & 1 & 6 \\
$v_{12}(\mathrm{~Hz})$ & $4.488 \times 10^{14}$ & $4.483 \times 10^{14}$ \\
$A_{12}\left(\mathrm{~s}^{-1}\right)$ & $2.36 \times 10^{5}$ & $1.07 \times 10^{7}$ \\
\hline \hline
\end{tabular}

Integrating over laser frequency yields the line-integrated target species density

$$
\int n d x=8 \pi \frac{g_{1}}{g_{2}} \frac{v_{12}^{2}}{A_{21} c^{2}} \int S(v) d v,
$$

where $n$ is the target species density; $g$ is the level degeneracy of the initial, 1, and final, 2, states; $v_{12}$ is transition (absorption) frequency; and $A_{12}$ is the Einstein coefficient for the transition. The values for the argon ion and neutral transitions used in this work are given in Table I.

When scanning across an absorption line, the target species sees Doppler shifted light based on the various particle velocities. The overall width of an absorption line is affected by several mechanisms including, but not limited to, natural linewidth, Stark broadening, power broadening, collisional broadening, Doppler broadening, and Zeeman broadening. ${ }^{19}$ For the magnetic fields (in the measurement region), gas pressures, and plasma densities used in this work, only Doppler broadening plays a significant role. However, it is important to remember that the reflected laser beam travels in the opposite direction to the injected beam. Therefore, any bulk flows along the laser direction are only resolvable if the thermal spread of the target species is much smaller than the bulk velocity of the target species. If the thermal spread is comparable to the bulk flow, the Doppler shifts in each direction will overlap and the measured velocity distribution will be artificially broadened. If the spatial distribution of the absorber is limited to a point source, as it is in Ref. 12, then it may be possible to resolve the true bulk velocity and temperature of the target species.

For Ar II measurements, the TOPTICA TA 100 diode laser used for LIF and CW-CRDS was tuned to 668.6138 $\mathrm{nm}$, corresponding to $3 \mathrm{~d}^{4} \mathrm{~F}_{7 / 2}-4 \mathrm{p}^{4} \mathrm{D}_{5 / 2}$ level transition in $\mathrm{Ar}$ II. The transition is comprised of circularly polarized sigma $(m= \pm 1)$ and linearly polarized $\pi(m=0)$ components. The laser is injected perpendicular to the magnetic field with its polarization axis aligned with the magnetic field to pump only the $\pi$ absorption lines. For a magnetic field strength of $1 \mathrm{kG}$ or less, the effect of the Zeeman splitting of the individual $\pi$ lines is much smaller than that of the Doppler broadening and is ignorable in the analysis. ${ }^{19}$ The LIF scheme used for Ar I is somewhat unconventional in that the initial $4 \mathrm{~s}\left({ }^{2} \mathrm{P}_{3 / 2}\right){ }^{1}$ state, the absorbing state, is not metastable. The initial state is populated by excitation from the ground state into nearby metastable states $4 \mathrm{~s}\left({ }^{2} \mathrm{P}_{3 / 2}\right)^{2}$ and $4 \mathrm{~s}\left({ }^{2} \mathrm{P}_{1 / 2}\right)$, followed by electron impact transfers from those states into the absorbing state. The initial state is pumped with a wavelength of $667.9125 \mathrm{~nm}$ into the $4 \mathrm{p}\left({ }^{2} \mathrm{P}_{1 / 2}\right)^{0}$ state. Keesee and Scime showed that because of its dependence on collisional mechanisms to populate the initial state, this Ar I LIF scheme is problematic at low, <10 mTorr, operating pressures. ${ }^{20}$ 


\section{EXPERIMENTAL APPARATUS}

The plasma source used for these measurements is the Compact Helicon for Waves and Instabilities Experiment (CHEWIE), shown in Figure 1. The device is a compact, vertically oriented helicon source. ${ }^{21,22}$ CHEWIE consists of a $60 \mathrm{~cm}$ long, $2.5 \mathrm{~cm}$ radius Pyrex $^{\text {TM }}$ glass tube connected to a $20 \mathrm{~cm}$ long, $7.5 \mathrm{~cm}$ radius stainless steel expansion chamber. The top of the glass tube defines $z=0$ and the center of the expansion chamber defines $r=0$. A $m=1$ half-turn right hand helical antenna wraps around the glass tube and is driven with steady-state RF power up to $1 \mathrm{~kW}$ or pulsed power up to $5 \mathrm{~kW}$ at frequencies between 10 and $16 \mathrm{MHz}$ to create and sustain the plasma. Two 140 turn water-cooled electromagnets in the source region produce a steady-state axial magnetic field of up to $1200 \mathrm{G}$. Nine radial ports in the expansion chamber provide diagnostic access to the plasma. Unless otherwise noted, all experiments presented here were conducted in the expansion region. Typical plasma parameters in the expansion region are $B=600-1200 \mathrm{G}, T_{e}=3-8 \mathrm{eV}, T_{i}=0.16 \mathrm{eV}, n$ $=1-10 \times 10^{10} \mathrm{~cm}^{-3}, \omega_{c i}=\sqrt{e B / m_{i} c}=2.2 \times 10^{5} \mathrm{rad} / \mathrm{s}, \rho_{i}$ $=V_{T i} / \omega_{c i}=0.4 \mathrm{~cm}, V_{T i}=\sqrt{k_{B} T_{i} / m_{i}}, \omega_{c e}=\sqrt{e B / m_{e} c}=1.6$ $\times 10^{10} \mathrm{rad} / \mathrm{s}, \rho_{e}=V_{T e} / \omega_{c e}=0.008 \mathrm{~cm}, V_{T e}=\sqrt{k_{B} T_{e} / m_{e}} \omega_{p e}$ $=\sqrt{4 \pi n e^{2} / m_{e}}=1.3 \times 10^{10} \mathrm{rad} / \mathrm{s}$, and $\lambda_{D}=\sqrt{k_{B} T_{E} / 4 \pi n e^{2}}$ $=7.8 \times 10^{-3} \mathrm{~cm}$, where $m_{i}$ is the ion mass, $m_{e}$ is the electron mass, $e$ is the electron charge, and $k_{B}$ is the Boltzmann's constant. Neutral gas pressure is measured with a Baratron gauge and the input gas rate is regulated with a MKS mass flow controller.

The TOPTICA diode laser consists of an external cavity diode laser seeding a tapered amplifier. The system produces up to $500 \mathrm{~mW}$ of power and has a mode hop free tuning range of $50 \mathrm{GHz}$. The $688.7 \mathrm{~nm}$ laser wavelength was chosen because it provides access to Ar I, Ar II, and He I absorption lines. ${ }^{23} \mathrm{~A} 60 \mathrm{~dB}$ optical isolator protects the diode laser system by eliminating back reflections from downstream optics. The wavelength of the laser light is continuously monitored with a high finesse WS7 UVII wavemeter. Before being steered into the plasma chamber with mirrors, the laser beam is modulated

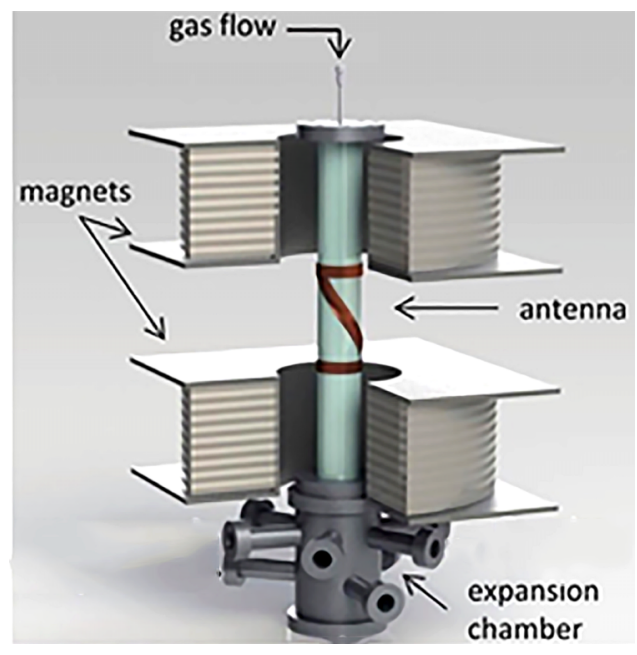

FIG. 1. The CHEWIE helicon plasma source. Shown here are the two water cooled electromagnets, the $m=1 \mathrm{RF}$ helical antenna, the plasma source tube, and the plasma expansion chamber with opposing and perpendicular ports for CW-CRDS and LIF. with an acousto-optic modulator (AOM) at $10 \mathrm{kHz}$. For ArII LIF, the fluorescent emission from the pumped excited state was collected and transported by a multimode optical fiber, passed through a $1 \mathrm{~nm}$ bandwidth filter centered around the fluorescent wavelength of $442.7 \mathrm{~nm}$, and measured by a narrow band, high-gain, Hamamatsu photomultiplier tube (PMT).

A classic CW-CRDS experimental configuration is shown schematically in Fig. 2. The ring-down cavity is formed by two highly reflective mirrors (>99.9\%). In a typical CW-CRDS system, one of the mirrors is placed on a piezo-electric transducer (PZT) so that the cavity length can be varied to bring the mirror separation into resonance as the laser wavelength is swept through the absorption line of the target species. When the resonance condition is achieved, evident by a dramatic increase in transmitted light out of the ring-down cavity, the incident light is blocked by the shutter. After the light is extinguished, the ring-down time in the cavity is measured.

Achieving the resonance condition with a narrow linewidth laser creates an additional experimental challenge. Resonance is achieved when a standing wave is formed in the cavity and constructive interference results in a significant gain in the transmitted intensity. For a pulsed laser with a wide linewidth, this condition is met with very little effort. For a narrow linewidth laser, a considerable effort is required to achieve the resonant condition and optimize the CW-CRDS system. In resonance, the cavity length is an integer number of half wavelengths, such that $L=n \lambda / 2$. The frequency separation of the longitudinal modes, $n$, is

$$
\delta v_{F S R}=c / 2 L,
$$

where $L$ is the length of a non-confocal cavity, $c$ is the speed of light, and $\delta v_{F S R}$ is the free spectral range of the cavity. Another key measure of cavity quality is the finesse, a measure of the cavity's ability to maintain a resonance condition. The higher the finesse, the harder it is to maintain a resonance condition. The finesse, $F$,

$$
F=\sqrt{\pi} R /(1-R),
$$

is directly related to the effective path length of the optical cavity, $L /(1-R)$, where $R$ is the reflectivity of the mirrors. The finesse of the cavity determines the sensitivity of the CW-CRDS diagnostic, and the higher the finesse, the higher the sensitivity. The finesse calculation assumes that the light injected into the cavity stays in the cavity. An "unstable" cavity is one in which light eventually bounces out of the cavity during successive round trips. The stability of a cavity created with focusing mirrors is defined by its length and the radius of curvature of the mirrors $r_{n}$. For stability, a cavity must satisfy

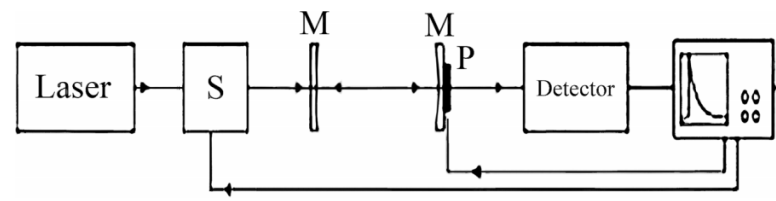

FIG. 2. A typical CW-CRDS experimental configuration. The shutter (S) blocks the laser beam, for CW lasers, once the light intensity in the cavity formed by the mirrors (M) exceeds a threshold level when a cavity resonance is achieved. The PZT (P) adjusts the cavity length for a CW-CRDS system to create a resonance. An example ring-down is shown on the oscilloscope. 
the condition ${ }^{24}$

$$
0<g_{1} g_{2}<1,
$$

where $g_{1}=1-L / r_{1}$ and $g_{2}=1-L / r_{2}$. In these experiments, both mirrors have the same radius of curvature and the stability condition becomes $r<L<2 r$ and $0<r<L$.

The full spectrum of cavity modes includes both longitudinal $(q)$ and transverse $(m, n)$ modes each with a frequency of

$$
v_{q m n}=\frac{c}{2 L}\left[q+\frac{n+m+1}{\pi} \cos ^{-1}\left(\sqrt{g_{1} g_{2}}\right)\right],
$$

where $q, m$, and $n$ are integers. The separation of longitudinal modes with the same $m$ and $n$ is $\delta \boldsymbol{v}_{F S R}$. Transverse modes with different $m, n$, but the same $q$ are conventionally labeled as $\mathrm{TEM}_{m n}$ modes. Each transverse mode has a distinct crosssectional intensity profile and travels along a unique path in the optical cavity. The reader is referred to Ref. 24 for a detailed discussion of non-symmetric cavities and the full spectrum of cavity modes.

For CW-CRDS, the external laser must couple to a single cavity mode, ideally the cylindrically symmetric, Gaussian intensity profile, $\mathrm{TEM}_{00}$ mode aligned along the optical axis of the cavity. Mode coupling requires matching the beam waist, intensity profile, and location of the focused injected beam at the focal point of the cavity. The beam waist in the cavity (see Fig. 3) is

$$
W_{0}=\sqrt{\frac{L \lambda}{\pi}}\left[\frac{g_{1} g_{2}\left(1-g_{1} g_{2}\right)}{\left(g_{1}+g_{2}-2 g_{1} g_{2}\right)^{2}}\right]^{1 / 4} .
$$

To create a Gaussian intensity profile from the diode laser, we coupled the $m=1$ order emanating from the AOM into a single mode optical fiber. The optical fiber provided the additional advantage of allowing us to place the laser far away from the helicon plasma source, which was a source of considerable RF noise and vibration. After exiting the optical fiber, the light was collimated with a single lens and then the beam size matched to cavity waist and focal point of the cavity with two additional convergent lenses. Theoretical calculations, assuming Gaussian beams and using ray transfer matrices, ${ }^{24}$ provided guidance for the focal lengths and positions of the lens. For a single thin lens of focal length $f$, an incident Gaussian beam of waist $W_{1}$ at a distance of $d_{1}$ from the lens converges to a waist of $W_{2}$ at distance $d_{2}$ after the lens where $d_{1}$ and $d_{2}$ are

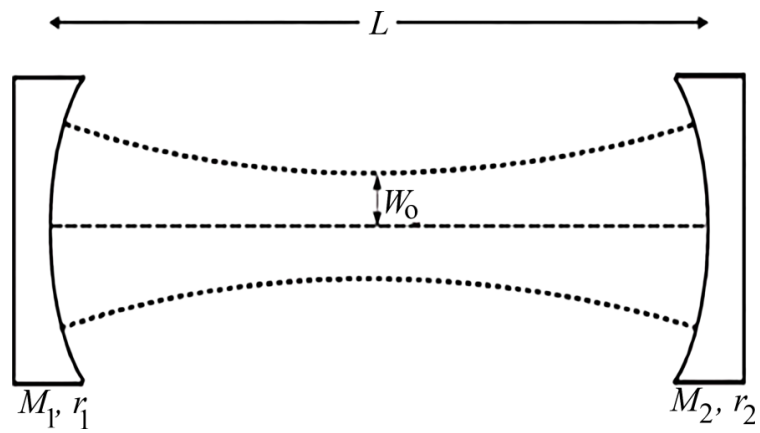

FIG. 3. Spatial profile of a $\mathrm{TEM}_{00}$ mode in a focused cavity. The beam waist of $W_{0}$ is shown at the focal point of the cavity. given by

$$
\begin{aligned}
& d_{1}=f \pm \frac{W_{1}}{W_{2}} \sqrt{f^{2}-f_{0}^{2}}, \\
& d_{2}=f \pm \frac{W_{2}}{W_{1}} \sqrt{f^{2}-f_{0}^{2}},
\end{aligned}
$$

where $f_{0}=\pi W_{1} W_{2} / \lambda$. Even after including corrections for the finite thickness of the lenses and the radius of curvature of the mirror that the injected beam must pass through, the final lens positions had to be adjusted by up to $10 \%$ to optimize the mode matching to the cavity.

Light transmitted through the cavity is measured with a New-Focus photodetector (Model-2051). The photo-receiver is a silicon diode photodetector with a built-in transimpedance amplifier and an adjustable bandwidth of up to $10 \mathrm{MHz}$. The signal from the photodetector is sent to a GAGE MAGMA 16-bit digitizer and a tracking circuit. The high sampling rate and 16-bit resolution of the GAGE digitizer were critical for obtaining high-quality ring-down decay curves. The tracking circuit is used to extinguish the laser light once an optimal resonance condition is achieved. The signal from the photodetector is compared to an adjustable DC threshold voltage. If the detector voltage exceeds the threshold voltage, the transistortransistor logic (TTL) pulse train modulating the AOM is modified so that for the next $80 \mu \mathrm{s}$, no diffraction pattern is created, effectively extinguishing the $m=1$ beam being injected into the ring-down cavity. The tracking circuit used for these experiments is improved over earlier versions by the addition of a buffer amplifier to prevent loading of the tracking circuit output by the low input impedance AOM. Custom software scanned the laser frequency, recorded wavelength measurements for each measured ring-down event recorded by the GAGE digitizer, and set the threshold voltage on the tracking circuit. Typical acquisition rates were several hundred ring-downs in a few minutes while scanning the laser frequency over $10 \mathrm{GHz}$. The previous version of this apparatus required hours to obtain a few dozen ring-down measurements. A typical ring-down measurement with an exponential decay curve fit is shown in Fig. 4.

During optimization, the $99.995 \%$ reflective mirrors with $1 \mathrm{~m}$ radii of curvature from CRD-OPTICS (mounted in kinetic

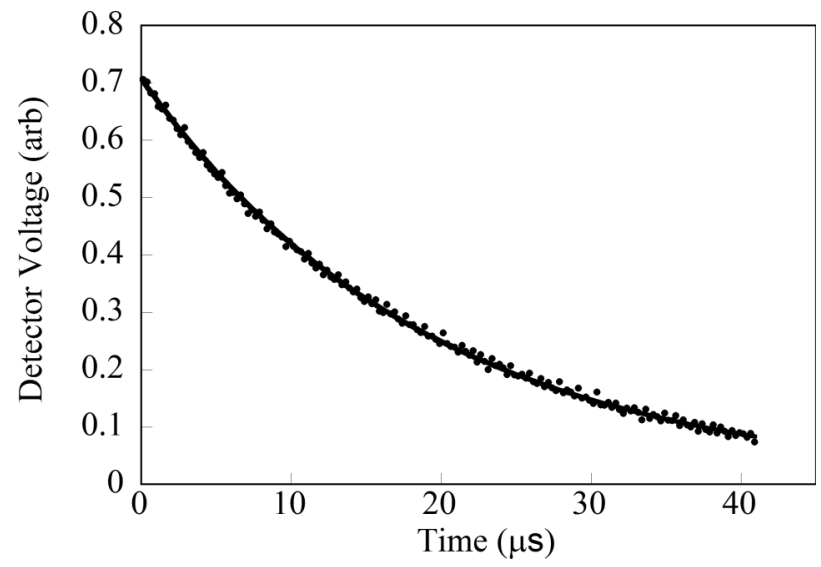

FIG. 4. Typical single ring-down measurement consisting of 8192 intensity measurements at a sample rate of $200 \mathrm{MS} / \mathrm{s}$. Only $2 \%$ of the measurements are shown for clarity. The solid curve is an exponential fit with a decay time of $19.2 \mu \mathrm{s}$. 

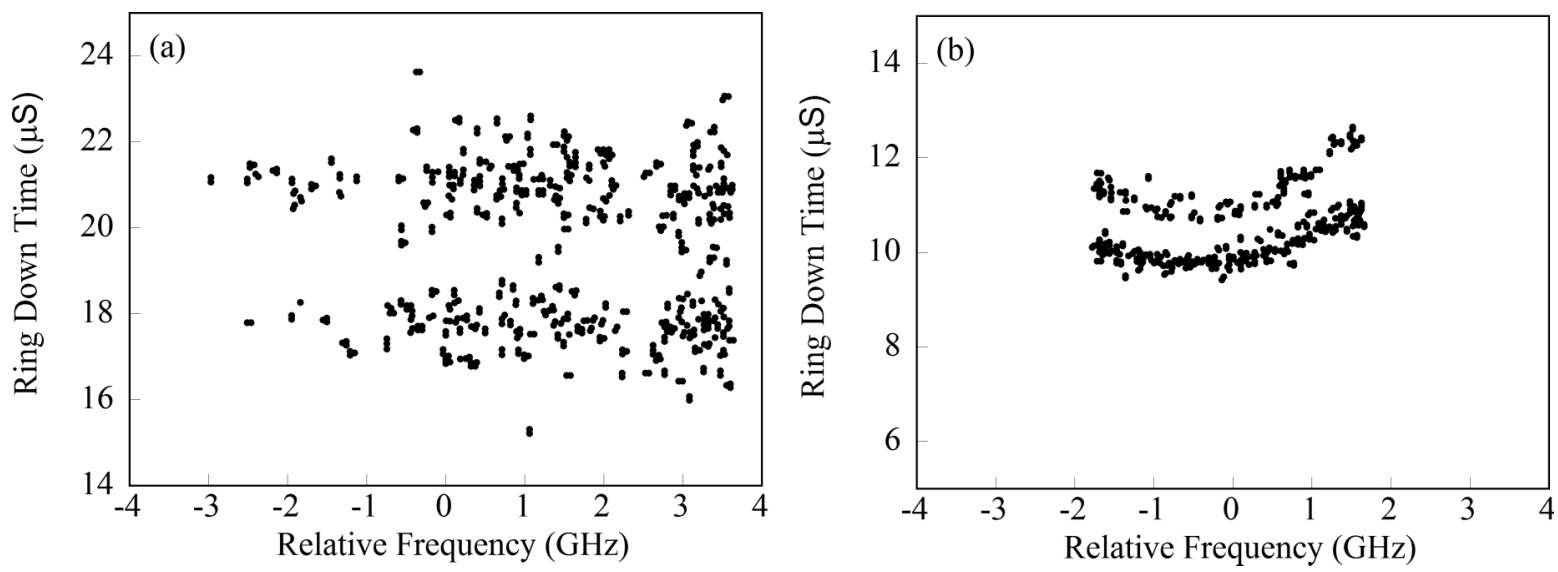

FIG. 5. Evidence of sub-optimal mode matching in ring down time measurements versus laser frequency relative to the center of the absorption line. Two different $\mathrm{TEM}_{\mathrm{mn}}$ modes with different characteristic ring-down times are present in (a) an empty cavity and in (b) a plasma filled cavity.

vacuum mounts also from CRD-OPTICS) and the coupling lenses are aligned and positioned to maximize the coupling to the $\mathrm{TEM}_{00}$ mode. If properly aligned, the intensity of the $\mathrm{TEM}_{00}$ mode resonance will exceed the intensities of higher order modes. Unfortunately, mechanical vibrations and thermal fluctuations will cause an optimized cavity to drift out of alignment over time and the intensities of the higher order modes may become comparable to the TEM 00 mode. In Fig. 5, two distinct distributions of ring-down times are evident. The threshold tracking circuit is unable to preferentially sample a single mode as a result of unoptimized mode matching during a scan through the absorption line. Note that every TEM $_{m n}$ mode has a different characteristic ring-down time $\tau$ due to its unique path through the cavity. It is possible to isolate specific modes post-acquisition, but only if they are as clearly distinguishable as in Fig. 5.

Another important factor in obtaining high-quality ringdown measurements is the extinction of the injected laser light once the threshold condition is achieved. If the injected light is not completely extinguished, higher order modes could be excited during a ring-down measurement or additional TEM $_{00}$ power injected. If either effects occur, the decay curve becomes a superposition of ring-downs and is no longer well fit with a single exponential decay. The extinction ratio of the AOM plays a pivotal role in extinguishing the light injected into the cavity. Some groups report that an extinction ratio $>75 \mathrm{~dB}$ is required to mitigate the effects of higher mode excitation during a ring-down. ${ }^{25}$ In Fig. 6, we shown histograms of ringdown times in an empty cavity using a single AOM operating at two different extinction ratios, $15 \mathrm{~dB}$ and $27 \mathrm{~dB}$. A common measure of the quality of a CRDS measurement is the normalized spread in average ring-down times,

$$
\text { error }=\frac{\delta \tau}{\bar{\tau}},
$$

where $\bar{\tau}$ is the averaged ring-down time and $\delta \tau$ is the standard deviation of the averaged ring-down times. There is a clear improvement in the ring-down time variance and distribution symmetry with the higher extinction ratio. Typically, CW-CRDS diagnostics operate with an error of $<1 \%$. For the measurements reported here, an IntraAction Corp. ATM803DA1 AOM with an extinction ratio of $45 \mathrm{~dB}$ was used.

Because of the transmission losses that would occur, it is not possible to obtain a resonance in the cavity if a glass window is placed between the two highly reflective mirrors. Therefore, it is necessary to place the highly reflective mirrors inside the CHEWIE plasma source to form the optical cavity. In helicon plasma sources, significant sputtering of the chamber walls occurs and the sputtered material accumulates on internal surfaces. In our original apparatus, deposition on
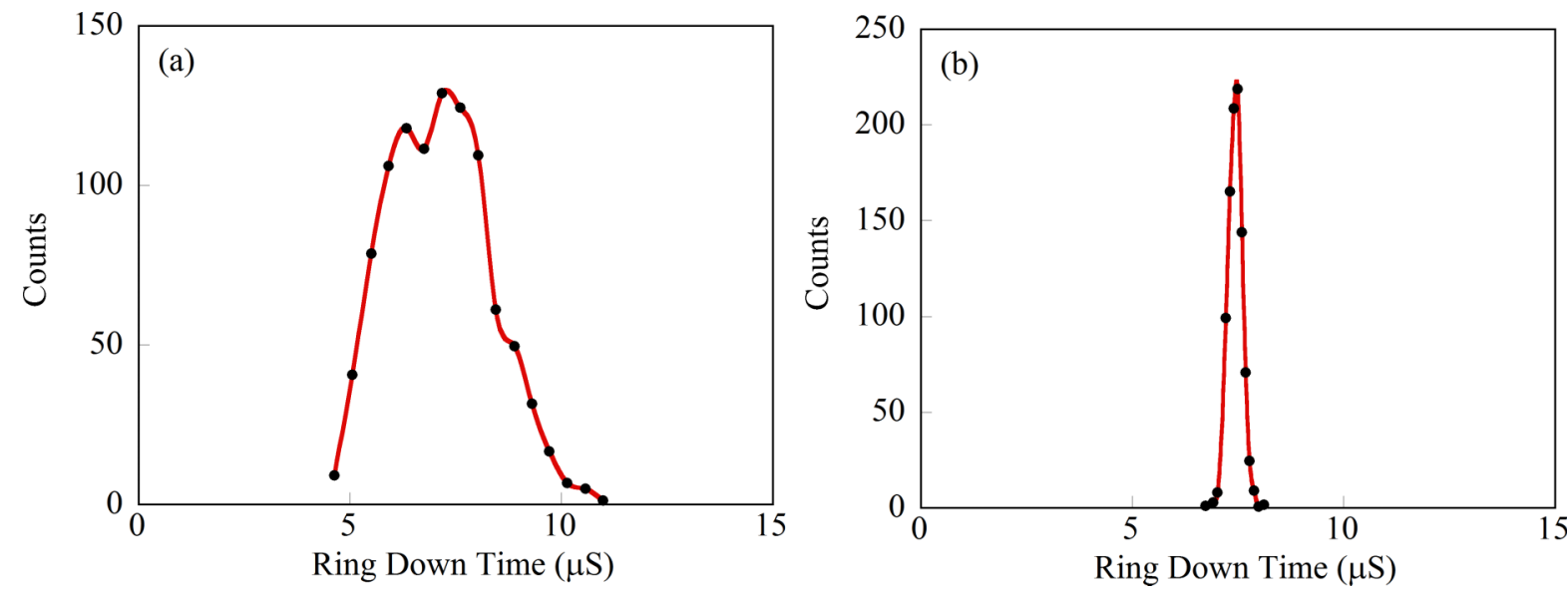

FIG. 6. Histograms of ring-down times obtained with an AOM with an extinction ratio of (a) $15 \mathrm{~dB}$ and (b) $27 \mathrm{~dB}$. 


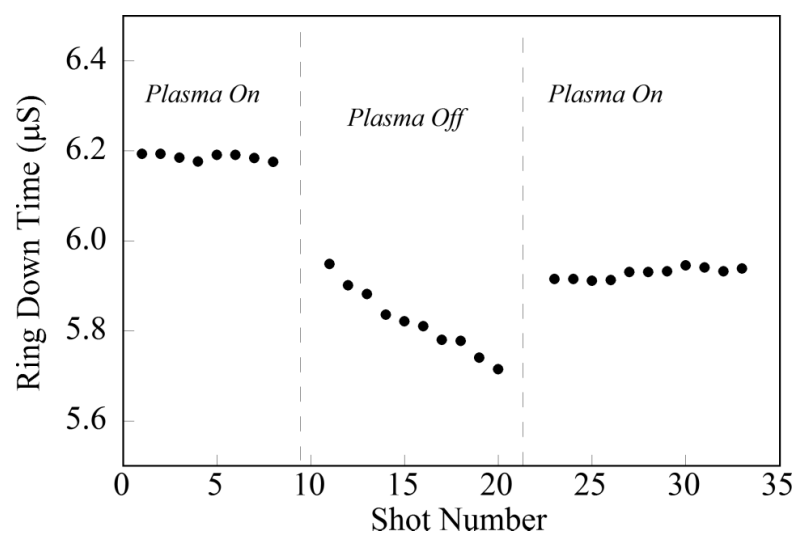

FIG. 7. Shot-to-shot variations in ring-down measurements due to particle deposition on the high reflectivity mirrors in the original CW-CRDS apparatus. ${ }^{1}$ The time between each measurement was approximately $5 \mathrm{~min}$.

the mirrors caused irreversible losses in reflectivity on the time scale of minutes (see Fig. 7). In these experiments, the effects of sputtering were completely eliminated by placing the mirror mounts on $25 \mathrm{~cm}$ long tubes mounted on opposite sides of the CHEWIE expansion chamber. Each tube included a gas feed port and the Conflat ${ }^{\mathrm{TM}}$ flanges at the ends of the tubes were mounted on the expansion chamber with a solid copper gasket. A $10 \mathrm{~mm}$ aperture was cut into each solid gasket and aligned with the optical axis of the cavity created by the mirrors. During plasma measurements, argon is fed in through the gas ports to create a "gas curtain" at the $10 \mathrm{~mm}$ aperture. These gas curtains successfully prevented particulates from depositing on the mirrors and runs of tens of hours exhibited no decrease in mirror reflectivity.

As noted previously, a key element in conventional CWCRDS systems is the mirror mounted on a PZT, which is used to vary the cavity length at each laser frequency to create a resonance. In our original apparatus, mechanical vibrations from the vacuum pumping system and the RF power supply were so large that it was nearly impossible to isolate $\mathrm{TEM}_{00}$ mode resonances. The PZT itself appeared to pick up RF noise and create additional instability in the system. For these experiments, the vacuum pumps were decoupled from the chamber

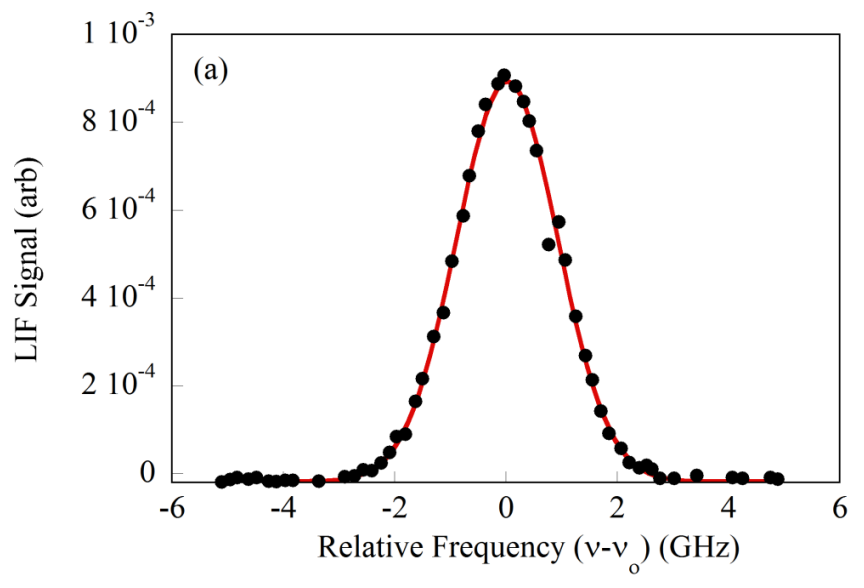

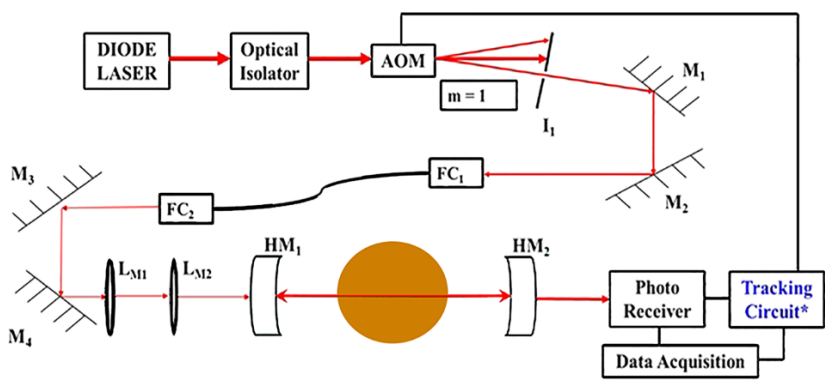

FIG. 8. Schematic of the CW-CRDS apparatus. Passing through the optical isolator, the diode laser is modulated with an acousto-optic modulator (AOM). The spatial filer, $I_{1}$, picks off just the $m=1$ beam from the AOM which is then coupled into a single mode optical fiber. The two mode matching lenses, $L_{\mathrm{M} 1}$ and $L_{\mathrm{M} 2}$, couple the laser light into the cavity formed by the two high reflectivity mirrors, $H_{\mathrm{M} 1}$ and $H_{\mathrm{M} 2}$, that surround the plasma. Light leaking out of the $H_{\mathrm{M} 2}$ mirror is recorded with the photodetector, and when a resonance occurs and the light intensity increases rapidly, the tracking circuit quickly shuts off the AOM and the decay in the cavity is recorded with the data acquisition system.

with a series of bellows and as many sources of vibration and electrical noise were eliminated. However, enough mechanical instability remained that without any scanning of the PZT the cavity would randomly develop a resonance at any laser frequency. Rather than try to eliminate the mechanical instability, we chose to take advantage of it and scan the laser more rapidly in frequency and over the same frequency range many times. Eliminating the PZT had the added benefit of removing the coupling of RF noise from the helicon source into the PZT and its controller. As the laser frequency is scanned many times over a fixed frequency range, the random vibrations bring the system into resonance and the threshold circuit activates, cutting off the injected laser light. Using this approach, the normalized standard deviation in CW-CRDS ring-down times for an empty cavity, for hundreds of ring-down measurements acquired over a few minutes for a range of wavelengths encompassing the absorption line, was less than $2 \%$.

The final version of the CW-CRDS apparatus is shown in Fig. 8. As shown, the $m=1$ order of the diffracted light from the AOM is coupled into the single mode optical fiber. After the fiber, the light is collimated and passes through two mode matching lenses, separated by $49.5 \mathrm{~cm}$, with focal lengths of

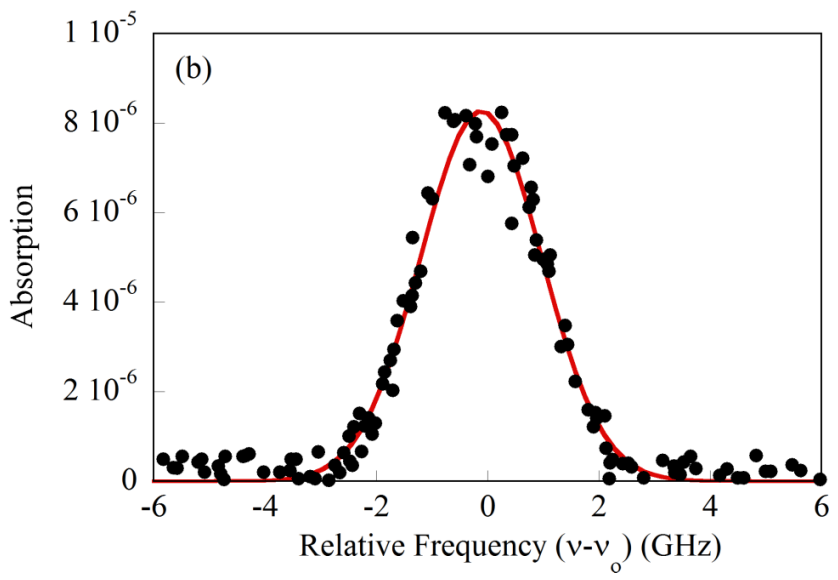

FIG. 9. Ar II velocity distributions obtained with (a) LIF and (b) CW-CRDS for $P_{\mathrm{RF}}=500 \mathrm{~W}, B=630 \mathrm{G}$, and a mass flow of 6 SCCM. The laser frequency is shown relative to the center of the absorption line. The resultant ion temperatures are $0.15 \pm 0.02 \mathrm{eV}$ and $0.22 \pm 0.02 \mathrm{eV}$, respectively. The metastable density obtained from the CW-CRDS measurement is $1.0 \times 10^{6} \mathrm{~cm}^{-3}$. 

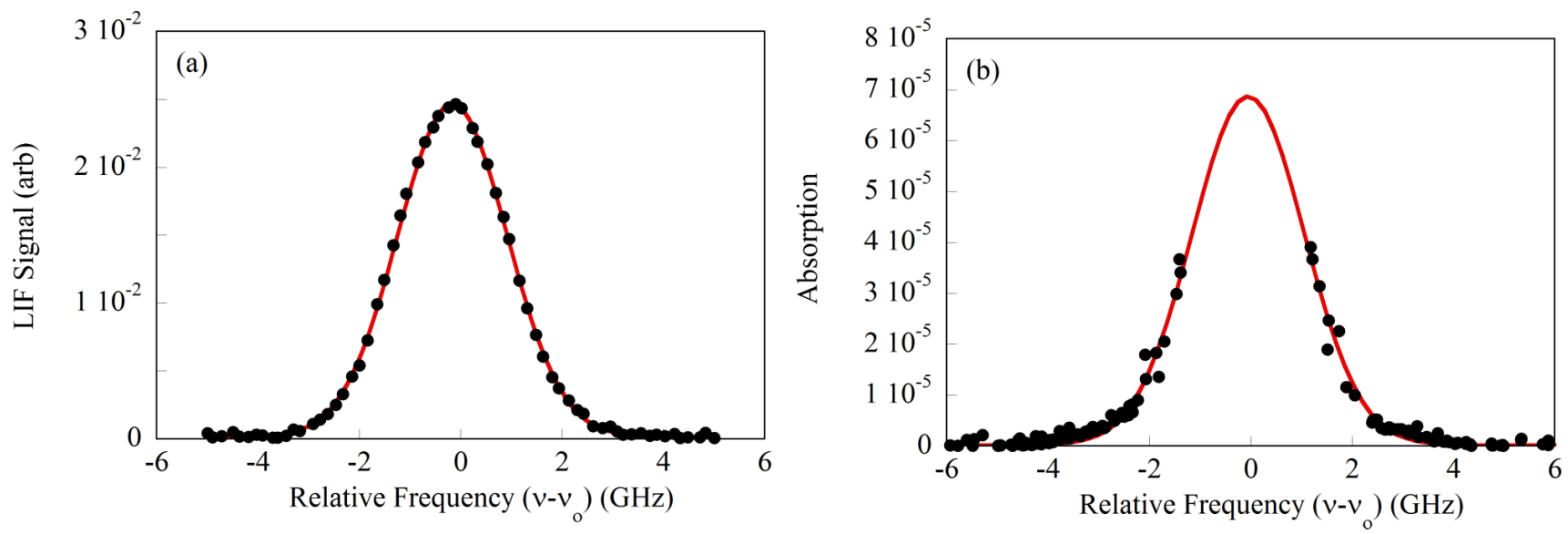

FIG. 10. Ar II velocity distributions obtained with (a) LIF and (b) CW-CRDS for $P_{\mathrm{RF}}=500 \mathrm{~W}, B=890 \mathrm{G}$, and a mass flow of 6 SCCM. The laser frequency is shown relative to the center of the absorption line. The resultant ion temperatures are $0.22 \pm 0.02 \mathrm{eV}$ and $0.23 \pm 0.02 \mathrm{eV}$, respectively. The metastable density obtained from the CW-CRDS measurement is $8.7 \times 10^{6} \mathrm{~cm}^{-3}$.

$30 \mathrm{~cm}$ and $15 \mathrm{~cm}$. The $15 \mathrm{~cm}$ focal length lens is positioned 81.32 from the center of the optical cavity to match the location and size of the injected beam to the $\mathrm{TEM}_{00}$ cavity mode. Using two mode matching lenses enabled the creation of a smaller beam waist in the cavity. The two lens matching system also enables better shaping of the injection beam and ultimately more efficient mode coupling.

\section{CRDS AND LIF MEASUREMENTS}

Shown in Fig. 9(b) is a typical CW-CRDS measurement of the Ar II VDF obtained for $500 \mathrm{~W}$ of RF power, a pressure of 8.4 mTorr, a mass flow rate of 6 SCCM (standard cubic centimeters per minute), and a magnetic field strength of $630 \mathrm{G}$. The constant input mass flow rate resulted in operating pressures ranging from 8.4 to $3.8 \mathrm{mTorr}$ as the magnetic field increased. For the same conditions, a LIF measurement of the Ar II VDF is shown in Fig. 9(a). Each point shown in the CW-CRDS plot is an individual ring-down measurement, effectively a snapshot of the plasma absorption at that wavelength on the time scale of a few microseconds. A Gaussian fit to the CWCRDS measurement yields a line-integrated ion temperature of $0.22 \pm 0.02 \mathrm{eV}$ and the fit to the LIF measurements yields an ion temperature of $0.15 \pm 0.01 \mathrm{eV}$. For the same conditions but for a magnetic field strength of $890 \mathrm{G}$, Ar II VDF

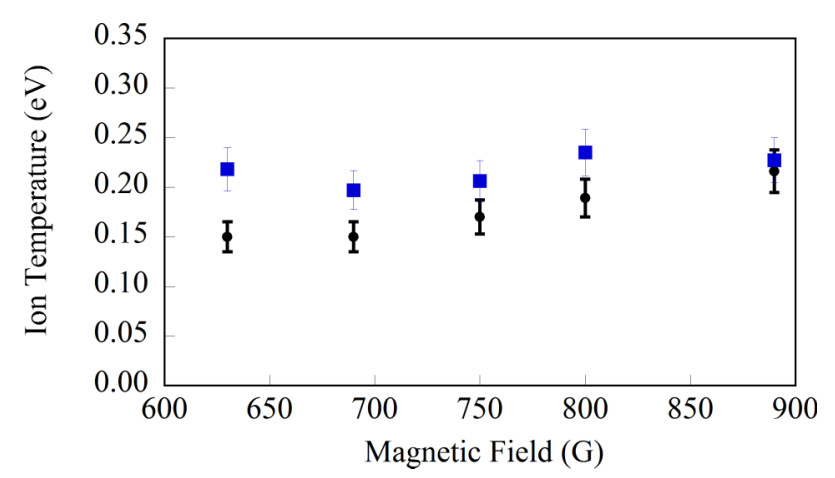

FIG. 11. Ion temperatures from LIF (circles) and CW-CRDS (squares) as a function of magnetic field strength for source parameters of $P_{\mathrm{RF}}=500 \mathrm{~W}$ and a mass flow of 6 SCCM. measurements obtained with CW-CRDS and LIF are shown in Fig. 10. At the higher field strength, the fit to the CWCRDS data yields the same ion temperature of $0.23 \pm 0.02 \mathrm{eV}$ while the fit to the LIF data yields an ion temperature of $0.22 \pm 0.01 \mathrm{eV}$. The absence of measurement points near the peak of the CW-CRDs IVDF measurement results from a dramatic decrease in transmitted laser power near the peak of the absorption line due to the substantial increase in the density of the metastable state. In other words, the CW-CRDS method is so sensitive to the metastable state population that nearly all the injected light is being absorbed when the state density gets large.

While the differences between the LIF and CW-CRDS temperature measurements for argon ions are statistically significant at the lower magnetic field strength, it is important to remember for any comparison of CW-CRDS and LIF measurements that LIF is a spatially resolved technique, whereas CW-CRDS integrates the absorption over the entire laser path length (and therefore superimposes any bulk drifts smaller than then thermal spread in velocities as noted before). Therefore, a slightly hollow ion temperature profile, as is often observed in helicon sources, ${ }^{26,27}$ could easily explain the difference in calculated ion temperatures. Another very likely possibility is that weak radial ion flows on the order of the tens of meter per second artificially broaden the CW-CRDS

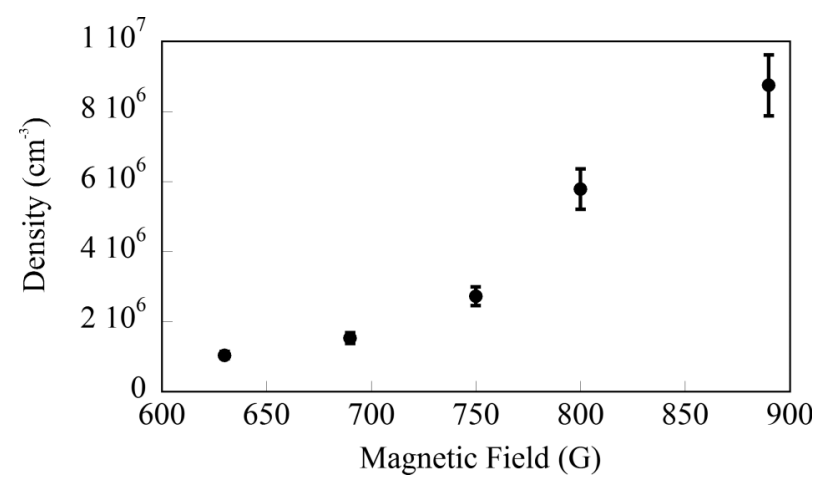

FIG. 12. Absolute metastable Ar II density calculated from CW-CRDS versus magnetic field strength for $P_{\mathrm{RF}}=500 \mathrm{~W}, B=890 \mathrm{G}$, and a mass flow of 6 SCCM. 
line shape. Since the laser light reflects back and forth over the entire cavity many times, any bulk radial drifts will contribute to peaks in the measured VDF on both sides of the rest frame frequency (red and blue Doppler shifts), thereby creating a broader line-integrated VDF. Similar magnitude radial flows have been seen in previous LIF measurements in other helicon sources. ${ }^{28}$ The ion temperatures obtained from CW-CRDS and LIF over the entire magnetic field strength scan are shown in Figure 11. The CW-CRDS ion temperatures are consistently higher than those obtained by LIF but well within the range seen in similar argon helicon plasmas. Measurements of the initial metastable argon ion state absolute density are shown in Fig. 12. The CW-CRDS measurements indicate a sharp increase in Ar II metastable density, a factor of more than four, for this range of magnetic field strengths. Nearly identical scaling of the plasma density versus magnetic field strength in CHEWIE was observed in krypton helicon plasmas. ${ }^{21}$

Shown in Fig. 13 is a sequence of LIF and CW-CRDS measurements originating from the neutral argon $4 \mathrm{~s}\left({ }^{2} \mathrm{P}_{3 / 2}\right)^{1}$ state obtained for magnetic field strengths ranging from 630 to $860 \mathrm{G}$, a RF power of $300 \mathrm{~W}$, and a mass flow rate of 5 SCCM (resulting in an operating pressure range of 12.8-6.3 mTorr as the magnetic field increased). Also shown in Fig. 13 are Gaussian fits to each of the measured neutral VDFs. The most obvious feature is the absence of LIF measurements of the neutral VDF below $800 \mathrm{G}$. Below $800 \mathrm{G}$, the LIF signal is indistinguishable from the background light. These magnetic fields correspond to operating pressures below 10 mTorr, the same operational limit for Ar I LIF seen by Keesee and Scime. ${ }^{20}$ However, the much more sensitive CW-CRDS
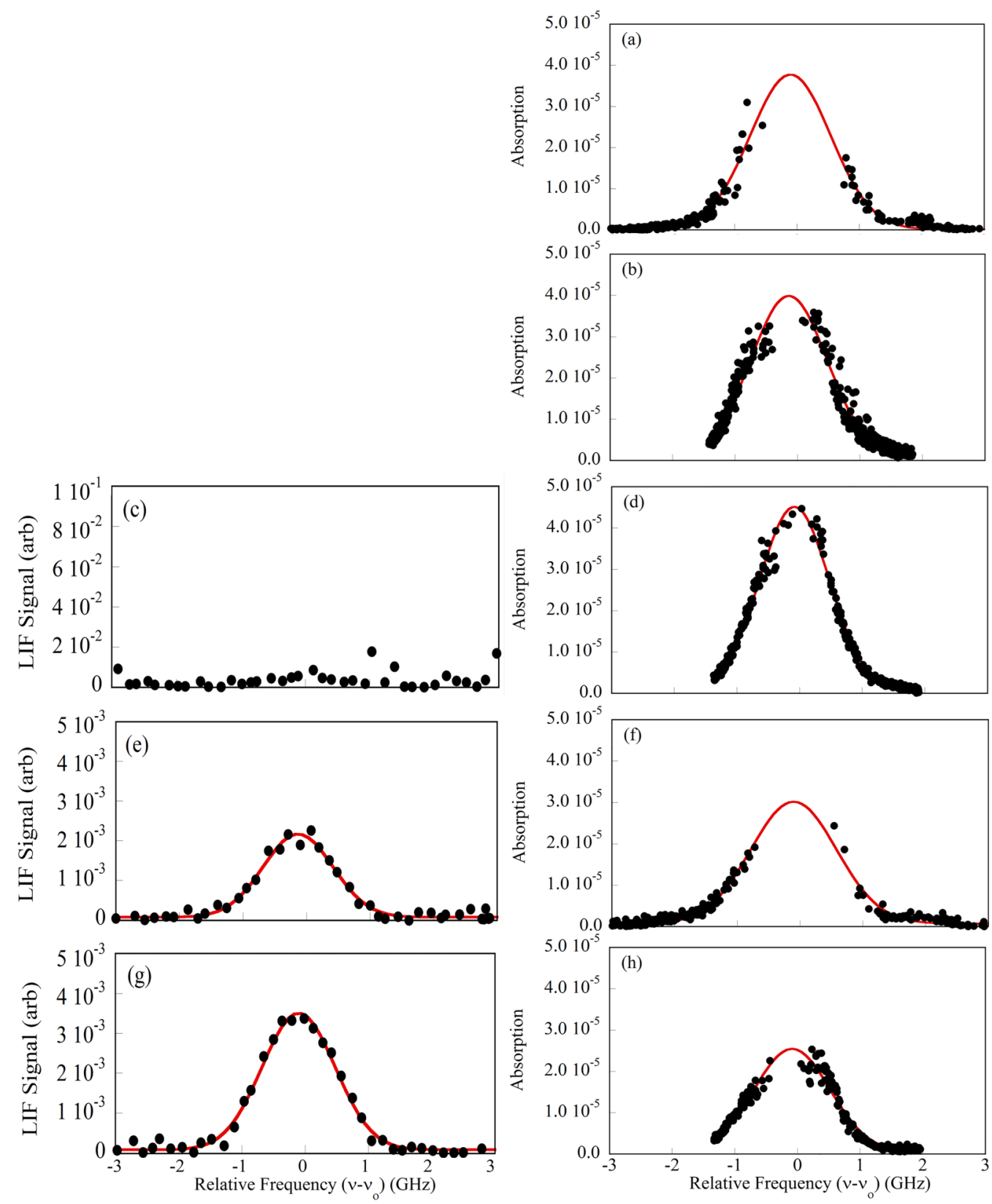

FIG. 13. Argon neutral VDFs for $P_{\mathrm{RF}}=300 \mathrm{~W}$ and a mass flow of $5 \mathrm{SCCM}$ obtained by (a) CW-CRDS at $630 \mathrm{G}$, (b) CW-CRDS at $690 \mathrm{G}$, (c) LIF at $750 \mathrm{G}$, (d) CW-CRDS at $750 \mathrm{G}$, (e) LIF at $800 \mathrm{G}$, (f) CW-CRDS at $800 \mathrm{G}$, (g) LIF at $860 \mathrm{G}$, (h) CW-CRDS at $860 \mathrm{G}$. The laser frequency is shown relative to the center of the absorption line. 
method provides high quality VDF measurements at all the magnetic field strengths investigated.

\section{DISCUSSION}

These measurements demonstrate it is possible to perform in situ CW-CRDS measurements of argon neutral and argon ion VDFs in a high-density, helicon plasma source. The measurement apparatus is robust, functions in a high vibration environment and is resistant to the effects of sputtering in the plasma source. The CW-CRDS VDF measurements have been validated through LIF measurements of the same initial state. For those plasma source conditions that are inaccessible by LIF for specific species, e.g., argon neutrals at pressures below 10 mTorr, the CW-CRDS technique provides VDF measurements with excellent signal to noise.

Some of the CW-CRDS measurements, particularly at source parameters that yield the largest absorbing state densities, have few if any, data points near the peak of the absorption line. The lack of data around the peak of the VDF affects the quality of the fits used to extract temperature, flow, and absolute density values. The data gap occurs because there are so many absorbers, and thus, the intensities of the mode never exceed the threshold voltage on the tracking circuit and ringdown measurements are not triggered. If the threshold voltage is lowered to measurements around the peak absorption, the threshold circuit no longer exclusively selects the $\mathrm{TEM}_{00}$ mode for measurement and the resultant ring-down measurements include a superposition of multiple modes. An improvement to the CW-CRDS technique that would eliminate this problem is to implement a programmable, adaptive threshold level during data acquisition.

In addition to the mode threshold issue, we noticed significant saturation around the absorption peak for certain plasma conditions (see Fig. 14). Saturation occurs when the target state is depopulated by continuous excitation by high intensity laser light. Absorbers near the natural absorption frequency (nearly at rest) effectively see twice the intensity of the laser light during cavity resonance (compared to the absorption in the wings). Thus, the region around the peak is most to

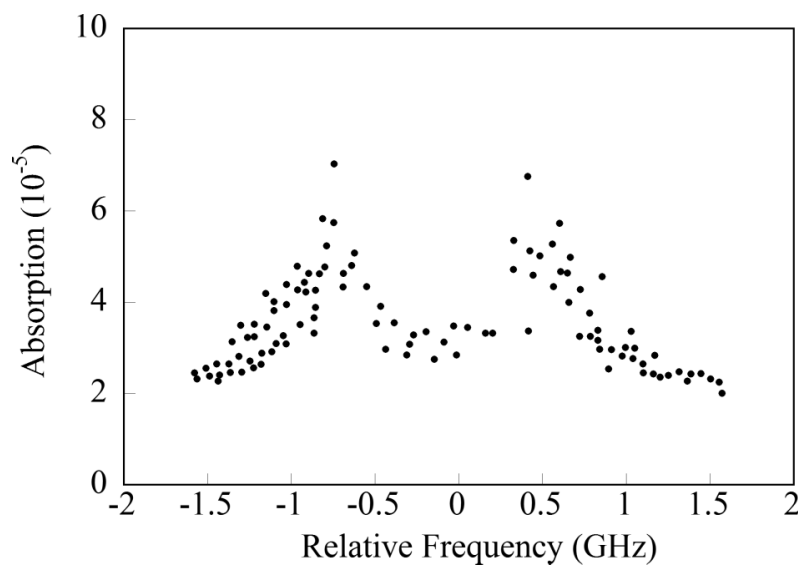

FIG. 14. Measured absorption versus laser frequency relative to the center of the absorption line. Argon neutral CW-CRDS measurement obtained at $P_{\mathrm{RF}}=300 \mathrm{~W}$ and $B=630 \mathrm{G}$. The middle of the measurement shows clear evidence of saturation. exhibit saturation. Such effects have been observed in other CW-CRDS measurements. ${ }^{29}$ Figure 14 shows an extreme case of a narrow frequency scan (where the laser is near the natural absorption frequency for the majority of the measurement) and the majority of absorbers near the natural absorption frequency have been depleted. In this case, the plasma source conditions were such that the density of neutral metastable states was likely very low. In less extreme cases, the peak of the distribution may only have a slight dip or appear noisy. The result is an underestimate of the density and artificially inflated temperatures obtained from the width of the distribution. Saturation effects are avoidable with a lower intensity laser, which requires an improved high-gain, high-bandwidth photodetector.

Surprisingly, the CW-CRDS approach was most effective when the experimental hardware was greatly simplified. The diode laser beam was shaped to match the Gaussian nature of the $\mathrm{TEM}_{00}$ mode in the cavity with a simple single-mode optical fiber. Injection of the probe beam into the cavity was accomplished with a simple two-lens mode-matching system and intrinsic vibrations of the plasma source were used to bring the cavity into resonance with the laser across the entire laser frequency scan. One tremendous advantage of the $\mathrm{CW}$ CRDs technique is the ability to measure the absolute density of the absorbing species without need of calibration. These measurements are ideally suited for validating collisional radiative models of plasma sources and such comparisons are yet to be made in the literature.

Finally, the most important implication of this work is that the CW-CRDs diagnostic approach developed for these experiments can, with the appropriate light source and mirrors, be utilized for any plasma species with a natural absorption line. Plasma species without readily accessible LIF schemes have defied precise velocity distribution measurements for years. This CW-CRDS diagnostic technique will work for any plasma species with an absorption line accessible by a tunable laser.

\section{ACKNOWLEDGMENTS}

This work is supported by the U.S. National Science Foundation (NSF) Grant No. PHY-1360278. We thank M. Umair Siddiqui for helpful comments on the manuscript and S. Chakraborty Thakur for extensive advice and guidance during the measurements.

${ }^{1}$ S. C. Thaskur, D. McCarren, J. J. Carr, and E. E. Scime, Rev. Sci. Instrum. 83, 10D708 (2012).

${ }^{2}$ A. O'Keefe and D. A. G. Deacon, Rev. Sci. Instrum. 59, 2544 (1988).

${ }^{3}$ K. W. Busch and M. A. Busch, ACS Symposium Series (Oxford University Press, DC, 1999), Vol. 720.

${ }^{4}$ G. Berden, R. Peeters, and G. Meijer, Int. Rev. Phys. Chem. 19, 565 (2000).

${ }^{5}$ B. Paldus and A. Kachanov, Can. J. Phys. 83, 975 (2005).

${ }^{6}$ M. Mazurenka, A. J. Orr-Ewing, R. Peverall, and G. A. D. Ritchie, Chem. Phys. Lett. 101, 100 (2005).

${ }^{7}$ G. Berden and R. Engeln, Cavity Ring-Down Spectroscopy: Techniques and Application (Wiley, UK, 2009).

${ }^{8}$ D. Z. Anderson, J. C. Frish, and C. S. Masser, Appl. Opt. 23, 1238 (1984).

${ }^{9}$ D. Romanini, A. A. Kachanov, N. Sadeghi, and R. Stoeckel, Chem. Phys. Lett. 264, 316 (1997).

${ }^{10}$ J. T. Hodges, L. P. Looney, and R. D. van Zee, Appl. Opt. 35, 4112 (1996).

${ }^{11}$ J. T. Hodges, L. P. Looney, and R. D. van Zee, J. Chem. Phys. 105, 10278 (1996). 
${ }^{12}$ A. P. Yalin and V. Surla, Opt. Lett. 30, 3219 (2005).

${ }^{13}$ N. Srivastava and C. Wang, J. Appl. Phys. 110, 053304 (2011).

${ }^{14}$ L. Tao, A. P. Yalin, and N. Yamamoto, Rev. Sci. Instrum. 79, 115107 (2008),

${ }^{15}$ B. C. Lee, L. T. W. Huang, N. Yamamoto, A. D. Gallimore, and A. P. Yalin, Rev. Sci. Instrum. 85, 053111 (2014).

${ }^{16}$ P. Sahay, S. T. Scherrer, and C. Wang, Rev. Sci. Instrum. 83, 095109 (2012).

${ }^{17}$ R. A. Stern and J. A. Johnson, Phys. Rev. Lett. 34, 1548 (1975).

${ }^{18}$ R. Murphy, I. A. Biloiu, M. Spencer, R. Hardin, and E. E. Scime, Bull. Am. Phys. Soc. DPP05, GP1.00013 (2005); available at http://flux.aps.org/ meetings/bapsfiles/dpp05_program.pdf.

${ }^{19}$ R. F. Boivin, West Virginia University Plasma Physics Lab Report PL-050, EPAPS-E-PHPAEN-10-003306, 2003.

${ }^{20}$ A. M. Keesee and E. E. Scime, Plasma Sources Sci. Technol. 16, 742 (2007).

${ }^{21}$ R. M. Magee, M. E. Galante, N. Gulbrandsen, and E. E. Scime, Phys. Plasmas 19, 123506 (2012)
${ }^{22}$ M. E. Galante, R. M. Magee, and E. E. Scime, Phys. Plasmas 21, 055704 (2014).

${ }^{23}$ A. M. Keesee, E. E. Scime, and R. F. Boivin, Rev. Sci. Instrum. 75, 4091 (2004).

${ }^{24}$ H. Kogelnik and T. Li, Appl. Opt. 5, 1550 (1966).

${ }^{25}$ H. Huang and K. K. Lehmann, Appl. Phys. B: Lasers Opt. 94, 355 (2009).

${ }^{26}$ J. L. Kline, E. E. Scime, R. F. Boivin, A. M. Keesee, X. Sun, and V. Mikhailenko, Phys. Rev. Lett. 88, 1950 (2002).

${ }^{27}$ E. E. Scime, J. Carr, Jr., M. E. Galante, and R. M. Magee, Phys. Plasmas 20 , 032103 (2013).

${ }^{28}$ E. E. Scime, R. Hardin, A. M. Keesee, C. Biloiu, and X. Sun, Phys. Plasmas 14, 043505 (2007)

${ }^{29}$ P. Macko, D. Romanini, and N. Sadeghi, Proc. Front. Low Temp. Plasma Diagn. IV 1, 223 (2001); available at http://www.gbv.de/dms/tib-ub-hannover/ 349515530.pdf. 